\title{
Spectral replacement using machine learning methods for continuous mapping of Geostationary Environment Monitoring Spectrometer (GEMS)
}

\author{
Yeeun Lee ${ }^{1}$, Myoung-Hwan Ahn ${ }^{1}$, Mina Kang ${ }^{1}$, Mijin Eo ${ }^{1}$ \\ $5 \quad{ }^{1}$ Department of Climate and Energy Systems Engineering, Ewha Womans University, Seoul, 03760, Republic of Korea \\ Correspondence to: Myoung-Hwan Ahn (terryahn65@ewha.ac.kr)
}

\begin{abstract}
Earth radiance in the form of hyperspectral data contains useful information on atmospheric constituents and aerosol properties. The Geostationary Environment Monitoring Spectrometer (GEMS) is an environmental sensor measuring such hyperspectral data in the ultraviolet and visible (UV/VIS) spectral range over the Asia-Pacific region. After successful

10 completion of the in orbit test of GEMS in October 2020, bad pixels are found as a remaining calibration issue to be updated with follow-up treatment. Currently, one-dimensional interpolation in the spatial direction is performed in operation to replace the erroneous pixels of GEMS, which causes high interpolation error for a wider defect area on a detector array. To resolve the issue, this study suggests machine learning methods with artificial neural network (ANN) and multivariate linear regression (Linear) to fill in a spectral gap of defective spectra. The machine learning models are trained with normal measurements to

15 emulate spectral relations between input and output radiances in a spectrum. For efficient training, dimensionality reduction for the input radiances is applied with principal component analysis (PCA) prior to the training process. The results show that the defect area at the wavelengths of strong absorption lines is better replaced with PCA-ANN with the error of 5\%, while PCA-Linear is better for reproducing radiances having strong correlation with input radiances. The shorter the spectral range of output radiances is, the smaller the prediction error is with PCA-Linear (0.5-5\%). Spectral and spatial discontinuity caused

20 by real bad pixels can be significantly improved with the trained machine learning models especially for wide defect areas. This study verifies that spectral relations of radiances in the UV/VIS spectrum are successfully reproduced with a simple machine learning model, which has high potential to be investigated further for enhancing measurement quality of environmental satellite measurements.
\end{abstract}

\section{Introduction}

25 Earth radiance contains useful information on the chemical composition in the atmosphere, especially when it is measured in the form of many contiguous spectral bands. This type of measurement is referred to as 'hyperspectral' (Goetz et al., 1985), because it is frequently sampled with high spectral resolution to accurately describe absorption lines of a targeted gaseous or particulate component (Boersma et al., 2004; Kang et al., 2020; Manolakis et al., 2019; Pan et al., 2017). The Geostationary Environment Monitoring Spectrometer (GEMS) on-board the Geostationary Korea Multi-Purpose Satellite-2B (GEO- 
30 KOMPSAT-2B) is an environmental sensor providing such a hyperspectral measurement in the ultraviolet and visible (UV/VIS) spectral region from 300 to $500 \mathrm{~nm}$ with a spectral resolution of finer than $0.6 \mathrm{~nm}$ (Kim et al., 2020). Following the launch of the satellite in February 2020, the in orbit test (IOT) of GEMS was successfully completed in October 2020 with some issues to be continuously monitored. The root cause of each issue is to be examined with collected long-term measurements, as it has been dealt with for other polar orbit sensors having similar sensor characteristics (Ludewig et al., 2020; Pan et al., 2019, 2020;

35 Schenkeveld et al., 2017).

One of the issues to be periodically monitored is about bad pixels, which refer to anomalous pixels having hot, cold, noisy or drifted readout values in raw data (Lo' pez-Alonso and Alda, 2002). The definition of bad pixels is not universal, and in this paper, it refers to all kinds of pixels presenting abnormal observation features. Bad pixel detection is based on sensor characterization by sorting out erroneous signals from normal trend. A few hot pixels were flagged as bad pixels during onground tests for GEMS and additional pixels have been sorted out during the IOT because of the impacts from the launch of the satellite and different environment conditions in space. The number of bad pixels tends to increase as time goes by (Kieffer, 1996), which indicates a significant number of bad pixels could affect to the measurement quality during the operation period of GEMS.

Following the bad pixel detection, replacement of measurements on bad pixel positions needs to be performed. There

45 are various ways to replace the measurements on bad pixels (Boldrini et al., 2012; Burger, 2009; Rankin et al., 2018), and in the GEMS calibration system, it adopts one-dimensional spatial interpolation on the detector (Fischer et al., 2007; Schläpfer et al., 2007). However, the approach showed its limitation during the IOT, when an area consisting of bad pixels is quite large and the adjacent pixels valid for spatial interpolation are too far from the erroneous area. Especially, when a scene on the earth dramatically changes, discontinuity caused by the interpolation becomes larger. This phenomenon affects to not only spatial

50 discontinuity on two-dimensional measurements, but also to a retrieval process using the spectral features contaminated by bad pixels (Marchetti et al., 2019).

In this respect, this study suggests machine learning methods to replace bad pixels on the radiance level using valid spectral features of normal measurements. As a way of replacement, we compare machine learning approaches using artificial neural network (ANN) and multivariate linear regression. Theoretically, it has been verified that ANN can accurately emulate 55 non-linear relations with a simple model structure when there are a large number of training data (Cybenko, 1989; Hornik et al., 1989). Machine learning methods have a high chance to successfully process hyperspectral data because the abundant datasets make training process more effective after breaking the curse of dimensionality with a proper pre-processing step (Gewali et al., 2018). Principle component analysis (PCA) is applied in this study, as it is useful to extract important information from hyperspectral measurements (Bajorski, 2011).

60 In remote sensing, the majority of researches on hyperspectral measurements has employed machine learning as a proxy of the radiative transfer model to retrieve geophysical state values with measured spectral radiances (Hedelt et al., 2019; Loyola et al., 2018; Zhu et al., 2018). There are fewer approaches applied to obtain radiation flux (Dorvlo et al., 2002; Zarzalejo et al., 2005) and even much fewer to obtain hyperspectral radiances for different purposes such as to accurately quantify 
radiative forcing in climate system (Taylor et al., 2016), increase spectral resolution (Le et al., 2020) and fill in a spectral gap for inter-calibration (Wu et al., 2018). A monochromatic radiance itself rarely contains any important meaning and thus seldom has it been a final target for machine learning. In this study, however, radiance at each wavelength of a targeted spectral region is a significant output for continuous mapping of GEMS measurements.

The following sections are organized as follows. Section 2 introduces sensor specification of GEMS including an overview of bad pixel detection and replacement methods of GEMS. In the section, a general description of machine learning models suggested in this study is introduced as well as the model structure and hyperparameter. Section 3 contains model optimization results and error analysis for wide defect regions. With the optimized model, bad pixel replacement is performed to real spectra contaminated by bad pixels and it is compared with linear interpolation, the operational method for replacing bad pixels of GEMS. In Sect. 4, conclusions are presented with limitations as well as further applications in the future study.

\section{Data and methods}

\section{$75 \quad 2.1$ Data description}

\subsubsection{GEMS}

GEMS is a UV/VIS imaging spectrometer in geostationary orbit observing the Asia-Pacific region $\left(5^{\circ} \mathrm{S}-45^{\circ} \mathrm{N}, 75^{\circ} \mathrm{E}-145^{\circ} \mathrm{E}\right)$ with high spatial and spectral resolution to retrieve key atmospheric constituents such as $\mathrm{O}_{3}, \mathrm{SO}_{2}, \mathrm{NO}_{2}, \mathrm{HCHO}$ and aerosol properties (Level 2) (Kim et al., 2020). The observation targets of GEMS are the Sun (irradiance mode) and the earth (radiance mode) and the description for each measurement mode is summarized in Table 1. GEMS observes the Sun on the purpose of calibration once a day with a premise of the measured solar irradiance being stable and nearly time independent. For earth measurements, GEMS scans the earth around 700 times within 30 minutes from east to west to cover the full field of regard (FOR) of GEMS. In both measurement modes, incident light from a scene passing through a fore-optics and spectrometer reaches to a two-dimensional detector array, the charge-coupled device (CCD) detector. The CCD of GEMS comprises 2,048 rows and 1,033 columns along the spatial direction from north to south $(\mathrm{N}-\mathrm{S})$ and the spectral direction with a sampling interval of $0.2 \mathrm{~nm}$, respectively.

Table 1 Top level measurement specifications of GEMS

\begin{tabular}{|c|c|c|}
\hline Measurement mode & Solar irradiance & Earth radiance \\
\hline $\begin{array}{c}\text { Data dimension } \\
\text { [spectral, spatial, scan] }\end{array}$ & {$[1033,2048]$} & $\begin{array}{c}{[1033,2048,695]} \\
\text { (nominal scene) }\end{array}$ \\
\hline Spectral range [nm] & \multicolumn{2}{|c|}{$300-500$} \\
\hline $\begin{array}{c}\text { Spectral sampling } \\
{[\mathrm{nm} / \text { pixel] }}\end{array}$ & \multicolumn{2}{|c|}{0.20} \\
\hline $\begin{array}{c}\text { Spectral resolution } \\
{[\mathrm{nm}]}\end{array}$ & $<0.60$ \\
\hline Spatial resolution $\left[\mathrm{km}^{2}\right]$ & - & $3.5 \times 8$ \\
\hline
\end{tabular}




\begin{tabular}{|c|c|c|}
\hline & & $($ spatial $\times$ scan $)$ \\
\hline $\begin{array}{c}\text { Measurement } \\
\text { frequency }\end{array}$ & Once a day & Every hour \\
$(13: 00$ UTC $)$ & $(00: 45-07: 45$ UTC $)$ \\
\hline
\end{tabular}

\subsubsection{Bad pixel}

Bad pixel detection is generally performed with dark-current measurements, which are taken without exposure to light for a certain integration time (Howell, 2006). Figure 1 illustrates bad pixel positions (in white) on the GEMS CCD detector array identified during the IOT. A cluster and distinct line shapes of bad pixels shown in Fig. 1a are initially identified during onground calibration before the launch of the satellite. Following the suggestions made by the instrument developers, linear interpolation along the spatial (N-S) direction is applied to replace the unusable measurements on bad pixel positions. With such a simple procedure, a single bad pixel could be properly substituted. However, it was found during the IOT that significant interpolation error could occur on the bad pixel positions denoted as Defect 1-3 (see Fig. 1b), especially when the spatial width of the invalid area is too wide as shown in Defect 2 and 3.

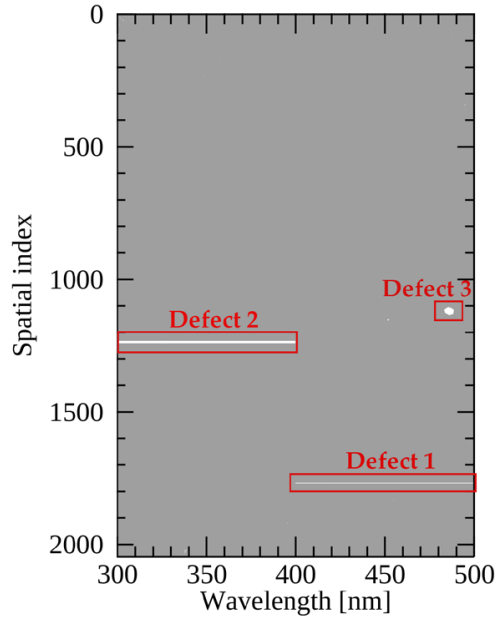

(a)
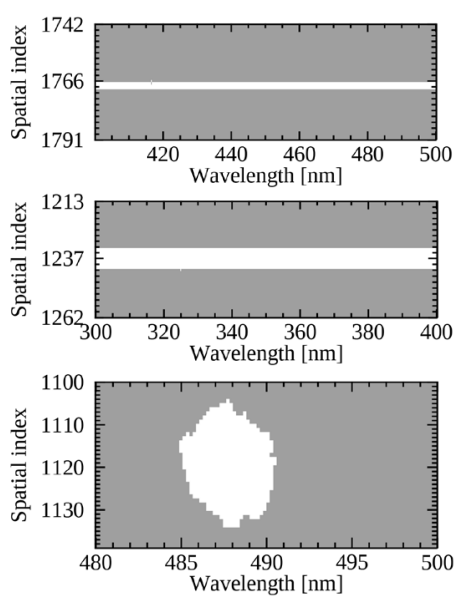

(b)

Figure 1 (a) Two-dimensional bad pixel map on the GEMS CCD detector along the spectral (x-axis) and spatial direction (y-axis) and (b) zooming in the bad pixel positions from top to bottom row for Defect 1-3. Bad pixels are marked in white.

This indicates the one-dimensional interpolation is ineffective to properly replace bad pixels for providing spatially continuous measurements. The interpolation error could also seriously affect to the Level 2 product of which the spectral fitting window is overlapped with a bad pixel area. For instance, cloud properties and aerosol effective height (AEH) of GEMS are retrieved from $\mathrm{O}_{2}-\mathrm{O}_{2}$ absorption bands around $477 \mathrm{~nm}$ (Choi et al., 2021; Kim et al., 2021) where the cluster of bad pixels is located (Defect 3). During the IOT, Defect 3 caused spatial discontinuity to the retrieved cloud and AEH distribution, which made the fitting window of the products modified to avoid bad pixel effects. Ozone properties are also affected by Defect 2

$105(300-400 \mathrm{~nm})$ as the spectral radiances within $300-380 \mathrm{~nm}$ are the major information for the retrieval of GEMS (Bak et al., 2019). 
To eliminate the spatial discontinuity, this study suggests machine learning methods to spectrally reproduce radiances on bad pixels instead of spatial interpolation as described in Fig. 2. The multivariate linear regression and ANN models are compared to evaluate model performance for reproducing earth radiance corresponding to the bad pixel positions of Defect 13. Solar measurements have high spatial homogeneity resulting in small interpolation error even on the large bad pixel areas, and are not considered in this study.

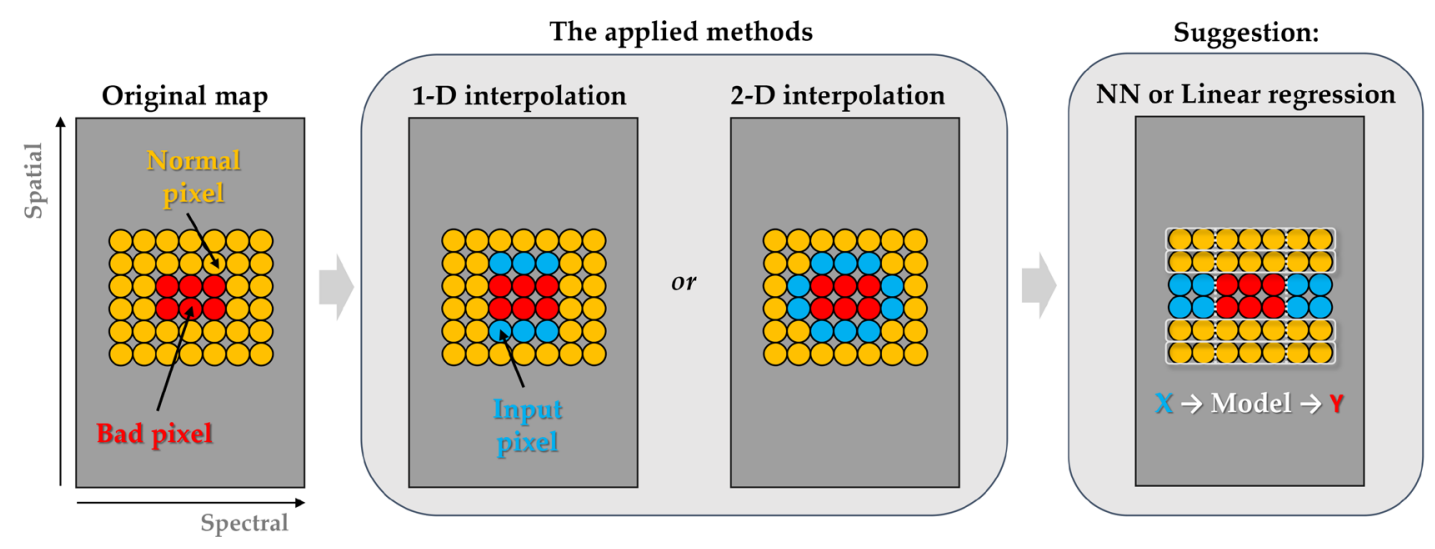

Figure 2 Schematic chart of the input (blue circle) and output pixels (red circle) on the GEMS CCD for the spatial interpolation and machine learning methods suggested in this study. Yellow circle indicates adjacent pixels to bad pixel position.

\section{$115 \quad 2.2$ Replacement approach}

\subsubsection{General description}

Reproduction of radiances on bad pixels is based on a fact that radiances at different wavelengths for a scene are highly correlated with each other (Liu et al., 2006; Wu et al., 2018). If the relations can be accurately established, some missing values in a spectrum could be properly represented with radiances at the other wavelengths. For training, radiance spectra of various scenes from clear sky to bright clouds can be obtained by randomly collecting measured spectra. To emulate the relations of the input and output radiances in a spectrum, the GEMS spectra are used which are measured on normal pixels located closer to a bad pixel area on the detector array. The basic premise of this approach is that neighbor pixels on the detector array would have similar measurement characteristics. After training a model with the normal spectra, a spectral gap caused by real bad pixels could be reproduced through the model with the rest of a spectrum as input parameters.

Because it is highly possible that input radiances have redundant information, PCA is applied for dimensionality reduction to compress the input radiances to low-dimensional principle components (PCs). The PCA process is given by the following Eq. (1):

$\mathbf{Z}_{n \times p}=\mathbf{X}_{n \times \lambda} \mathbf{W}_{\lambda \times p}$

where $\mathbf{Z}, \mathbf{X}$ and $\mathbf{W}$ represents the PC scores, input and PC matrix, respectively. The PC scores matrix $(\mathbf{Z})$ is obtained by projecting the input to the PC subspaces with $\mathbf{W}$, which is obtained by applying singular value decomposition to the $\mathbf{X}$. The 
subscript $n, \lambda$ and $p$ means the dimension of matrix corresponding to the number of datasets, wavelengths and the number of PCs, respectively.

With the compressed data, multivariate linear regression (PCA-Linear) and ANN (PCA-ANN) models are trained to define the relations between input $\left(\mathbf{X}_{\mathbf{m}}\right)$ and output $\left(\mathbf{Y}_{\mathbf{n}}\right)$ radiances in a spectrum. The PCA-ANN model is constructed with a simple feed-forward model with a hidden layer as described in Fig. 3. In the model optimization process, the PCA-ANN model with a hidden layer showed faster and more effective convergence of loss function than the models having multi-hidden layers in this study. For PCA-Linear, it adopts a simple linear model structure consisting of parameters such as weight and bias having the minimum mean squared error (MSE) between the regressed and measured radiances. After model optimization, it can be used to replace bad pixels $\left(\mathbf{X}^{\prime} \mathbf{m}, \mathbf{Y}^{\prime}{ }_{\mathbf{n}}\right)$ with radiances to be likely measured by the sensor.

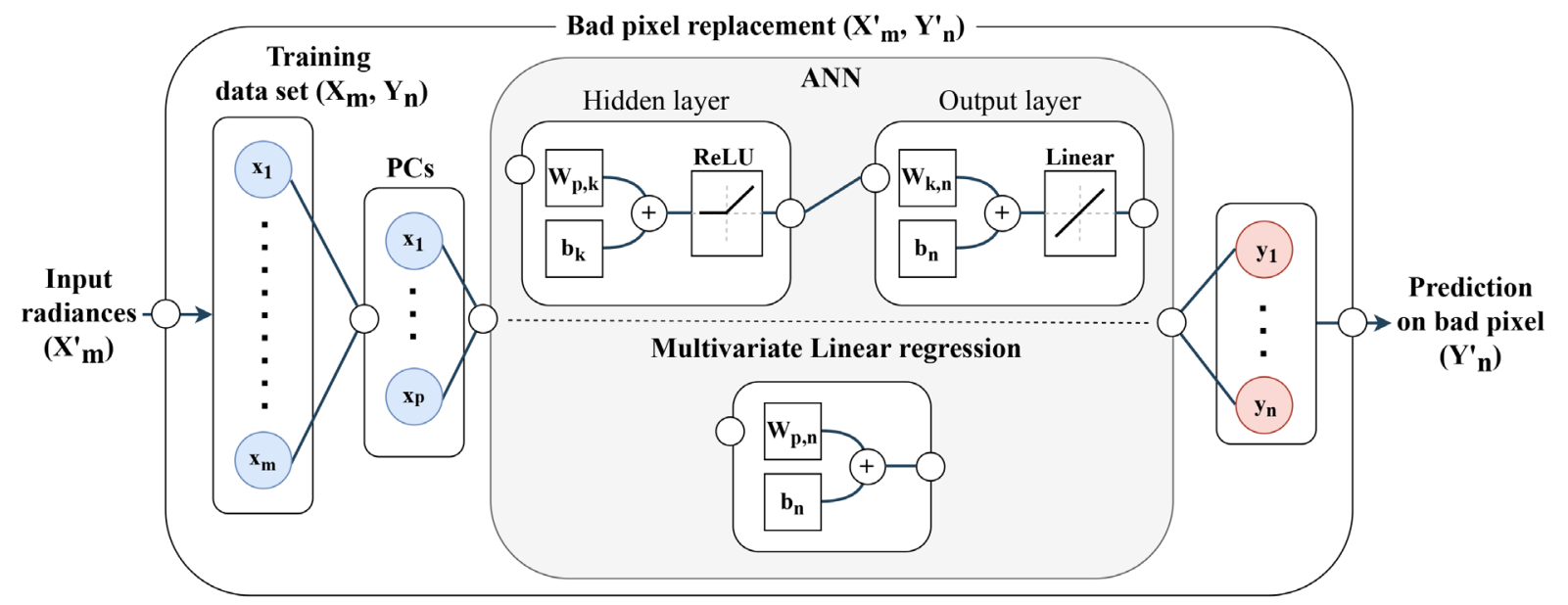

Figure 3 Schematic chart of the training and bad pixel replacement process. $\mathbf{W}$ and $\mathbf{b}$ represent weight and bias parameters in each layer. The subscript $m, n, p$ and $k$ is equal to the spectral dimension of input and output parameters, the number of PCs and hidden nodes of the ANN model, respectively.

\subsubsection{Input/Output and model optimization}

145 The input and output parameter as well as the optimized hyperparameter of the models are summarized in Table 2. Training and test data are constructed with GEMS radiance data measured in March-April 2021, which are randomly sampled out to update model parameter and check for overfitting, respectively. Input and output radiances are at the wavelengths with the spectral interval of $0.1 \mathrm{~nm}$ within the specified spectral ranges in Table 2. The spectral range of output radiances for Defect 13 is identical to each defect region and the rest part of a spectrum becomes input radiances. The solar zenith angle (SZA) and viewing zenith angle (VZA) are key variables determining the optical path of upwelling radiance, which are used as input variables together with radiances. The neural network constructed with the hyperparameter setting presented in Table 2 is implemented with TensorFlow, a high-level Application Programming Interface (API) written in Python. As described in Fig. 3, the activation function is the Rectified Linear Unit (ReLU) in the hidden layer of the ANN model. The structure itself is not complicated but it has multiple nodes in the input and output layers, which makes ReLU more competitive (Nwankpa et al., 
155 2018). The hyperbolic tangent (tanh) and sigmoid function show poor results especially when the output parameters have lower variance making the optimization stuck into the averaged value and preventing the model from being updated.

Table 2 Input and output (I/O) parameters for ANN training and hyperparameter for optimization of neural network.

\begin{tabular}{|c|c|c|c|c|c|}
\hline Model & Parameter & Defect 1 & Defect 2 & Defect 3 & Remark \\
\hline \multirow{3}{*}{$\mathrm{I} / \mathrm{O}$} & Input $\left(\mathrm{X}_{\mathrm{m}}\right)$ & $300-400 \mathrm{~nm}$ & $400-500 \mathrm{~nm}$ & $\begin{array}{c}460-483.9 / \\
491.1-500 \mathrm{~nm}\end{array}$ & $\begin{array}{c}\text { Random selection } \\
\text { (100,000 for } \\
\text { training and } \\
\text { test data) }\end{array}$ \\
\cline { 2 - 5 } & Output $\left(\mathrm{Y}_{\mathrm{n}}\right)$ & $400.1-500 \mathrm{~nm}$ & $300-399.9 \mathrm{~nm}$ & $484-491 \mathrm{~nm}$ & \\
\hline \multirow{3}{*}{$\begin{array}{c}\text { Hyper- } \\
\text { parameter }\end{array}$} & $\begin{array}{c}\text { Activation } \\
\text { function }\end{array}$ & \multicolumn{3}{|c|}{ ReLU } & \\
\cline { 2 - 5 } & Optimizer & \multicolumn{3}{|c|}{ Adam optimizer } & \\
\cline { 2 - 5 } & Loss function & \multicolumn{3}{|c|}{ Mean squared error } & \\
\cline { 2 - 5 } & Scaling & \multicolumn{3}{|c|}{ Standardization } & \\
\hline
\end{tabular}

For the optimizer, Adaptive Moment Estimation (Adam) is used which shows stable results compared to Stochastic Gradient Descent (SGD) and Root Mean Square Propagation (RMSProp) (Kingma and Ba, 2015). It is empirically found that SGD without gradient clipping tends to cause exploding gradient and RMSProp has difficulty reaching the global minima compared to Adam. Figure 4 presents the converging process of the PCA-ANN model for Defect 2 applying different optimizers. The model converges at 44, 98 and 33 epochs for Adam, SGD and RMSprop, respectively. Adam converges at the smallest MSE while the SGD converges with the highest MSE. RMSprop presents unstable loss for validation data and converges with higher MSE compared to Adam.

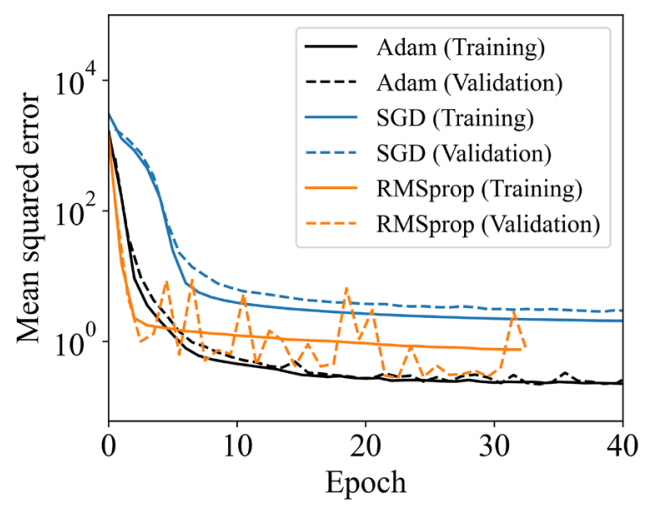

Figure 4 Loss function of training and validation data for Defect 2 with different optimizers such as Adam (black), SGD with the gradient clipping value of 0.5 (blue) and RMSprop (orange). 


\section{Results and discussion}

\section{$170 \quad 3.1$ Optimization results}

Earth radiance is determined by the interactions of light with trace gases, aerosols and clouds in the atmosphere and reflected properties of a scene. The magnitude of a spectrum is dominantly determined by the scene properties which result in strong linear relations among radiances in of a spectrum. In other words, when a scene is dark (bright), the upwelling radiances of the scene over the whole spectral region tend to become generally low (high). The PCA analysis performed for dimensionality reduction describes the characteristic with PC scores of input radiances in training data for Defect 2 (See Fig. 5). In the figure, it can be found that the first principal component (PC 1) is highly correlated with the magnitude of a spectrum represented by the radiance at $354 \mathrm{~nm}$. This indicates that strong linear relations among radiances in a spectrum are compressed to PC 1, which has the largest variance. The non-linear properties caused by atmospheric scattering, absorption, different optical paths and sensor noise are projected onto the other PC subspaces.

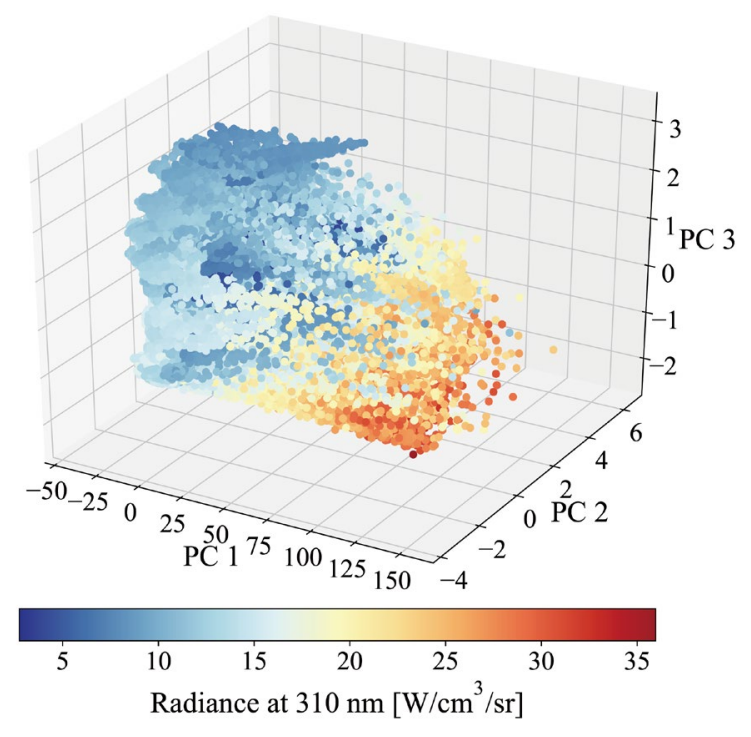

Figure 5 PC scores of input training data from 400 to $500 \mathrm{~nm}$ for Defect 2 after dimensionality reduction. Colorbar represents the radiance at $354 \mathrm{~nm}$ of output training data.

Figure 6 shows model optimization results depending on specific models and the number of PCs. Because the spectral range of output radiances differs for each defect region (Defect 1-3), model optimization needs to be performed separately.

185 The spectral range of output radiances for Defect 1 and 2 is wider than that of Defect 3 which results in higher MSE. PCAANN seems to be unstable for Defect 1 showing over-fitted features which might be caused by unfiltered outliers in output radiances of GEMS at the wavelengths longer than $480 \mathrm{~nm}$. It is empirically found that PCA-ANN is more vulnerable to outliers compared to PCA-Linear. Defect 2 is at the wavelengths where the upwelling radiances are largely affected by ozone, which increases non-linearity between input and output radiances. Because of the strong non-linearity, PCA-ANN shows better performance than PCA-Linear for Defect 2. Defect 3 has the smallest number of output parameters in a narrow spectral gap 
which causes strong correlation between input and output radiances. The loss functions (MSE) in Fig. 6c are small and converge quickly for both PCA-ANN and PCA-Linear models. With the results, the optimized number of PCs is set to 90 for all defect regions when loss functions for both training and test data efficiently converge, with PCA-Linear for Defect 1 and 3 and the PCA-ANN model for Defect 2.

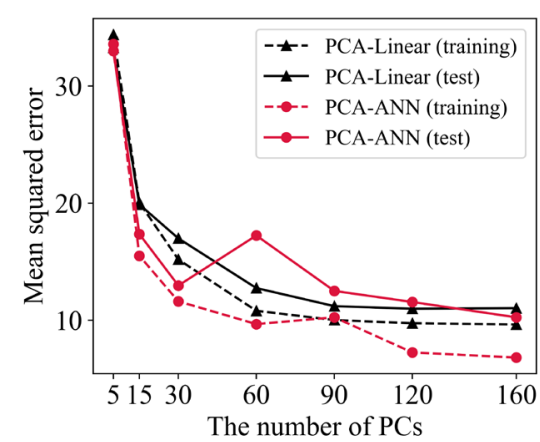

(a)

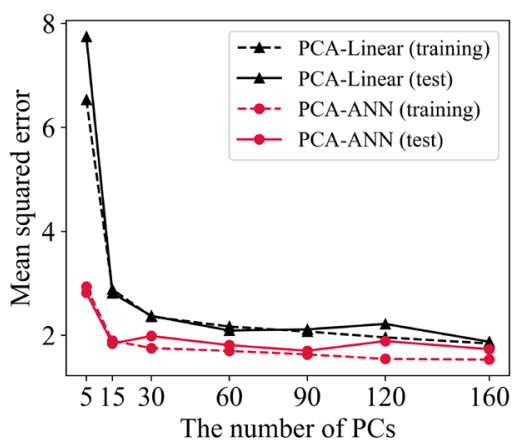

(b)

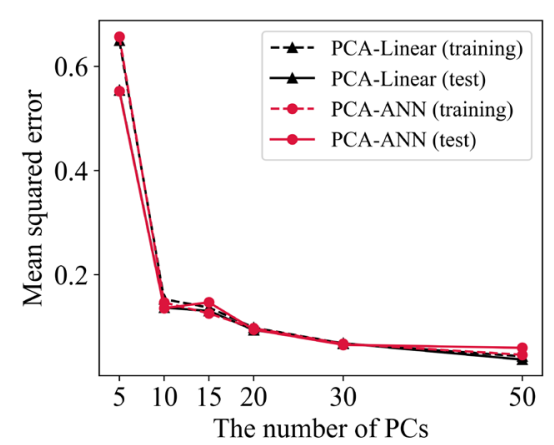

(c)

195 Figure 6 Loss function depending on the number of PCs with PCA-ANN (red) and PCA-Linear (black) model for predicting the output radiances corresponding to the spectral range of Defect 1-3 ((a): Defect 1, (b): Defect 2 and (c): Defect 3). The dashed and solid line indicates training and test results, respectively. The number of hidden nodes for ANN is double the number of PCs.

\subsection{Statistical evaluation}

The optimized model structures for Defect 1-3 are set as described in the previous section. Following that, in this section, model performance is statistically evaluated with training and test datasets specified in Table 2. Figure 7 presents mean and normalized root mean squared error (NRMSE) of the predicted output radiances with training and test data. The NRMSE is a statistical indicator normalized by mean radiance at each wavelength and it can be found that radiances affected by strong absorption lines have relatively high uncertainty. Especially, information from the radiances in 400-500 nm is insufficient to properly represent ozone absorption features at shorter wavelengths and it causes high uncertainty at the wavelengths shorter than $325 \mathrm{~nm}$ in Defect 2. It seems that the predicted radiances at shorter wavelengths are underestimated for both training and test data especially for dark scenes which have low signal-to-noise. This indicates it could be attributed to the insufficiently trained model parameters or the high measurement noise of dark scenes. Defect 3 has the lowest NRMSE because of strong linear relations between input and output radiances as previously mentioned in Sect. 3.1. The NRMSE is less than $0.1 \%$ for both training and test data over the Defect 3 spectral region. The results show that it is possible to successfully reproduce spectral features at a narrower spectral range with simple linear regression. 

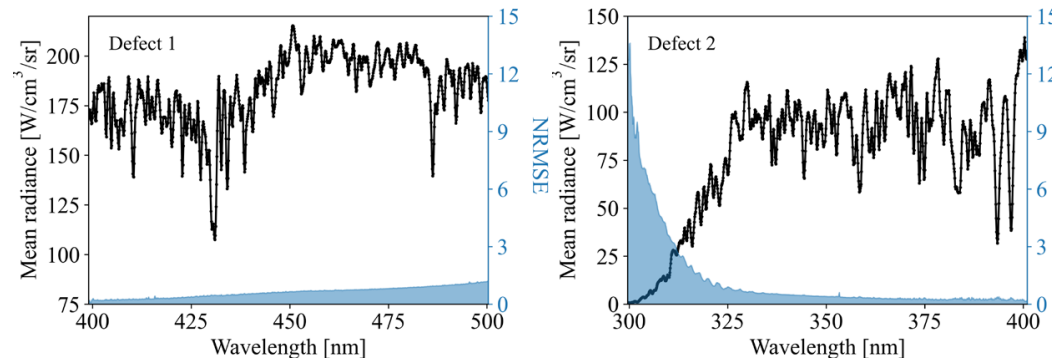

(a)
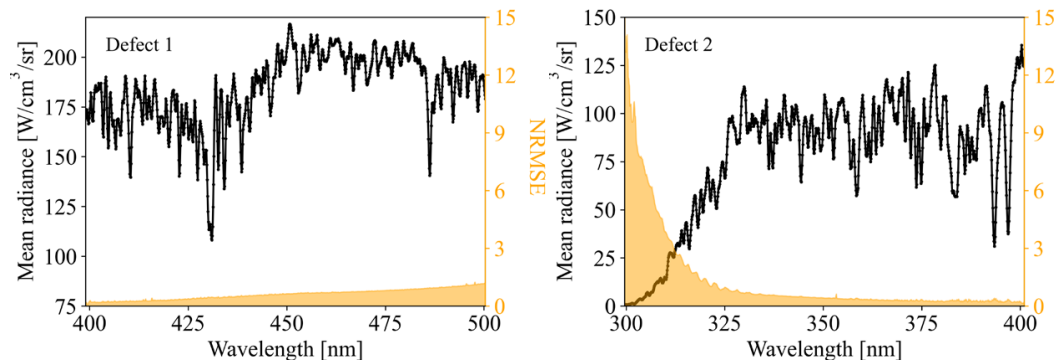

(b)
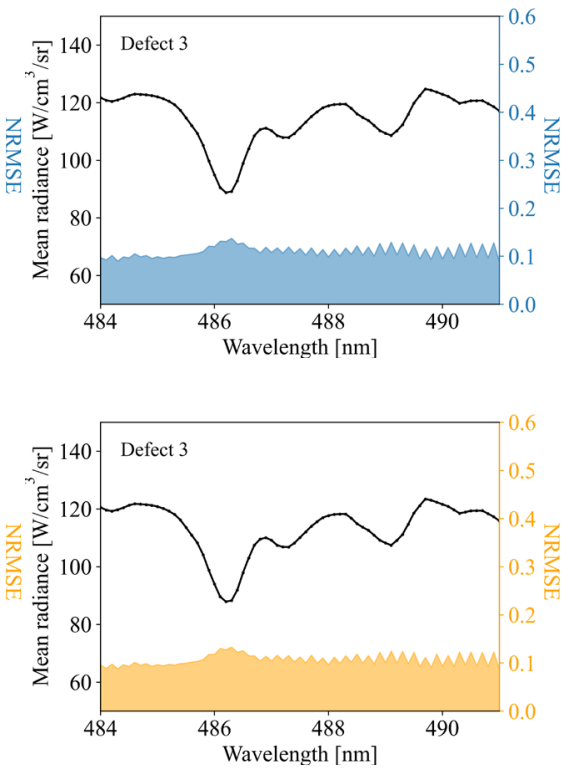

Figure 7 Mean (black) and NRMSE (blue) spectra of output radiances for Defect 1-3 with (a) training and (b) test datasets measured in March-April 2021. The unit of NRMSE is percent [\%].

Figure 8 shows the error histogram of each prediction model for Defect 1-3 with training and test data. The mode and mean of error histograms are on the order of 0.001-0.01 for test and training data. The machine learning models are good enough to properly emulate spectral relations between input and output parameters, but it is somewhat over-fitted to the training data causing a few outliers for the prediction of test data. Defect 2 has the largest standard deviation, which is consistent with the higher NRMSE at shorter wavelengths around $300 \mathrm{~nm}$ in Fig. 7. The largest kurtosis of Defect 2 for both training and test data indicates tails of the distributions are heavy compared to normal distribution, mostly from the radiances at shorter wavelengths. Considering that the overall prediction error is within 5\% except for the ozone absorption lines, the prediction models for Defect 1-3 are well constructed for further bad pixel replacement.

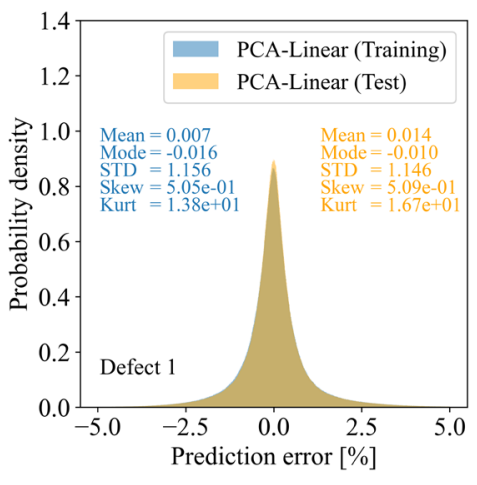

(a)

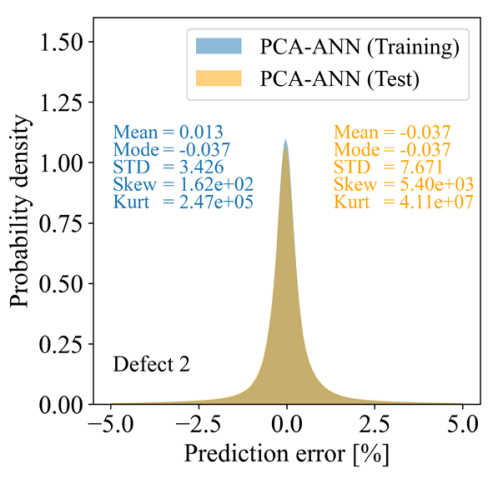

(b)

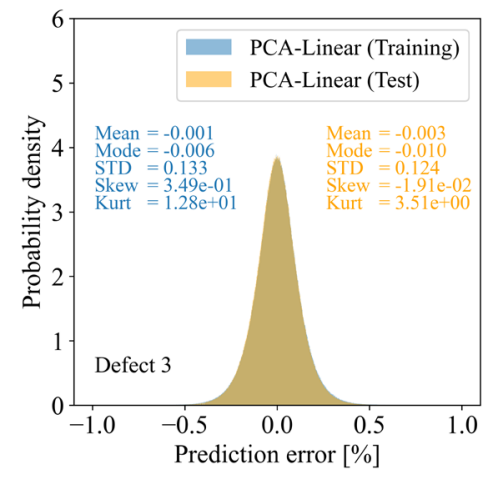

(c) 
Figure 8 Prediction error of randomly collected training (blue) and test (yellow) datasets measured in March-April 2021 with the optimized models for Defect 1-3 (PCA-ANN for Defect 2 and PCA-Linear for Defect 1 and 3). Prediction error is calculated with the difference between the predicted and measured radiances divided by the latter.

The training and test datasets presented in Fig. 7-8 are randomly collected spectra of GEMS measurements in MarchApril 2021, which guarantees a basic assumption in machine learning that the underlying population should be identical for training and test data (Zhen and $\mathrm{Li}, 2008$ ). However, in operation, the prediction model is obliged to be trained in advance with sufficient datasets for timely reproducing erroneous pixels of satellite measurements on a daily basis. This indicates the assumption might be violated if measurement characteristics of training and test data significantly change. To investigate further the effect, the prediction model is trained with training and test data measured in March and April 2021, respectively (see Fig. 9). The results show that histograms of test data for Defect 1-2 are more skewed than those of training data, when the measurement period of training and test datasets are separated. On the other hand, prediction for Defect 3 is independent to the measurement period of training and test data. This results indicate that the spectral features of GEMS spectra change profoundly as time goes by and the changes have strong spectral dependence within 300-500 nm.

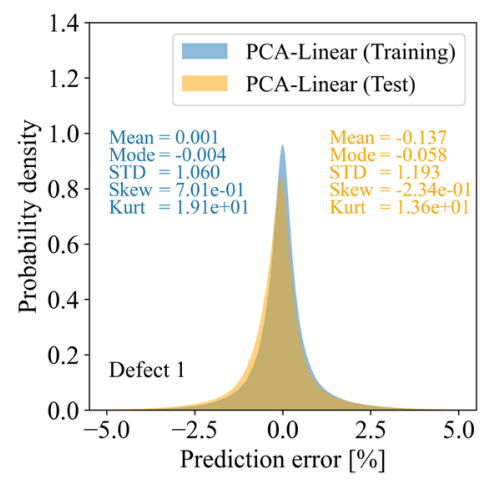

(a)

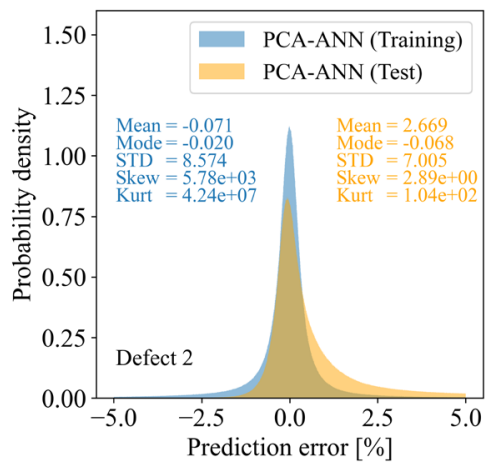

(b)

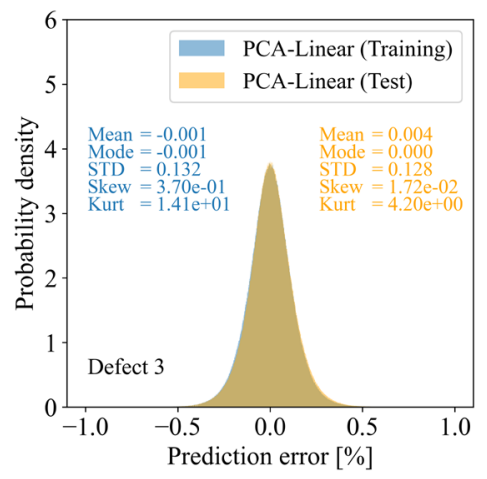

(c)

235 Figure 9 Same as Fig. 8 with training and test datasets measured in March and April 2021, respectively.

\subsection{Application to bad pixel replacement}

In this section, the prediction model trained with randomly collected spectra in March-April 2021 is employed for reproducing real bad pixels of Defect 1-3 from GEMS measurements on 2 May 2021. It is demonstrated with visual inspection of radiance images and quantitative comparison between machine learning methods and spatial interpolation, the current operational method for bad pixels in the GEMS calibration system. Figure 10 shows earth radiance images of GEMS on a particular region $\left(15^{\circ} \mathrm{N}, 102^{\circ} \mathrm{E}\right)$ affected by bad pixels of Defect 3, which has wider spatial width of bad pixels than that of Defect 1-2 along the N-S direction. Spatial discontinuity caused by bad pixels occurs as a horizontal line because GEMS scans the earth from east to west by sequentially recording scan images. Bad pixels of Defect 1-3 are stationary on the detector array, which causes measurements at certain latitudes unavailable. It is also noted that clouds are a main target making spatial discontinuity caused by bad pixels more noticeable with its high spatial variability. The spatial discontinuity is easily found in Fig. 10a because of 
the bad pixels inaccurately replaced with spatial interpolation. The PCA-Linear for Defect 3 shows better performance because the bad pixel positions easily found in Fig. 10a are entirely indistinguishable in Fig. 10b. The improvement is also found in Defect 2 (not shown), but with its narrower spatial gap compared to Defect 3, the improved results with PCA-ANN for Defect 2 is not visually recognizable. For Defect 1 , the spatial width of bad pixels along the N-S direction is narrower (two pixels width along the N-S direction) than that of Defect 2-3, which presents no significant difference between PCA-Linear and spatial interpolation.

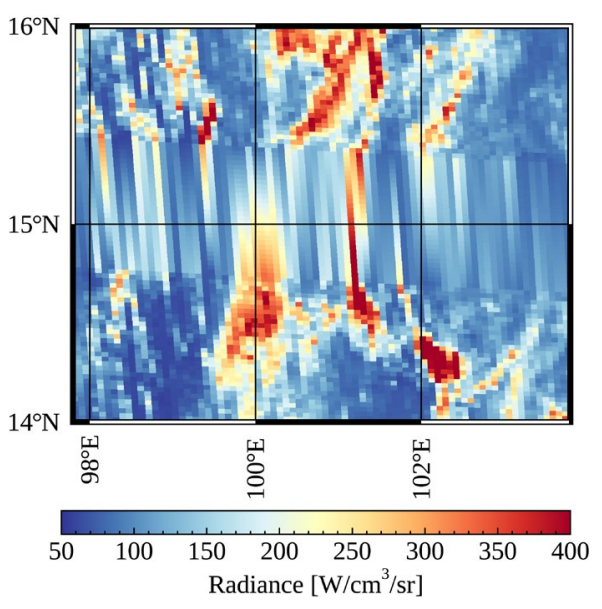

(a)

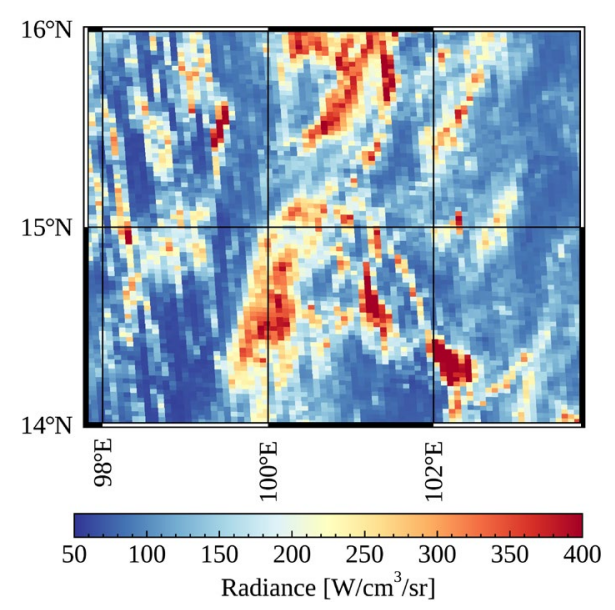

(b)

Figure 10 Comparison of bad pixel replacement between (a) spatial interpolation and (b) the machine learning method for the radiance at $487 \mathrm{~nm}$ for Defect 3 measured on 2 May 2021 (03 UTC).

A closer inspection is performed to analyze the reproduced spectral features for Defect 2-3. In spectral analysis, the effectiveness of bad pixel replacement methods could be well demonstrated at the wavelengths where the input and output radiances are met. If the replacement is successful, the partly replaced spectrum should have continuous spectral features over the whole spectral range. The spectral range affected by bad pixels for each defect region is $300-400 \mathrm{~nm}$ and $484-491 \mathrm{~nm}$, which corresponds to the range of output radiances for Defect 2-3, respectively. The rest part of a spectrum is input parameters for each defective region. As previously mentioned in Sect. 3.1., PCA-ANN shows better performance for Defect 2, while PCA-Linear is better for Defect 3 and thus each method is used for Defect 2 and 3, respectively. Figure 11 shows one of radiance spectra of Defect 2 and 3 from the GEMS measurement on 2 May 2021. For Defect 3 as shown in Fig. 11b, the reproduced spectrum with spatial interpolation shows totally unrealistic features compared to that with PCA-Linear because of the wider spatial gap of Defect 3. For Defect 2, however, the reproduced spectra with spatial interpolation and PCA-ANN show reasonable spectral features and the spectral discontinuity is not clearly discernible at the wavelength of $400 \mathrm{~nm}$. 


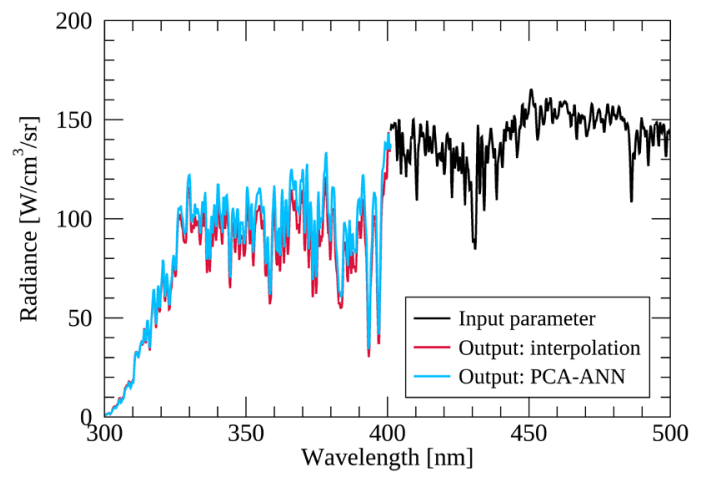

(a)

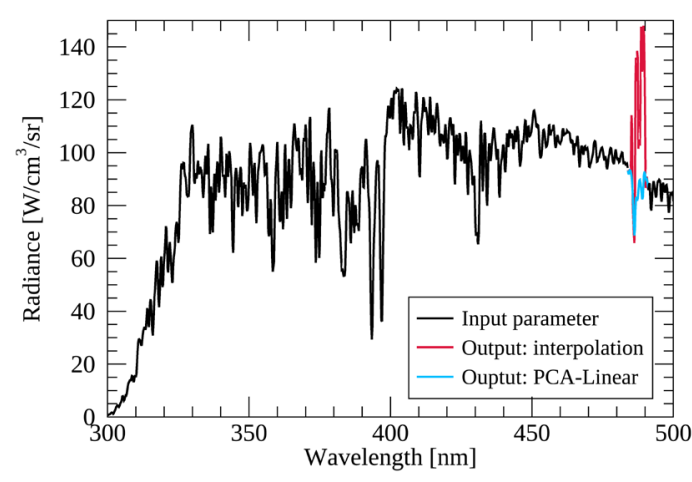

(b)

265 Figure 11 Radiance spectrum affected by bad pixels of (a) Defect 2 and (b) Defect 3 measured on 2 May 2021 (03 UTC). Bad pixel replacement is performed with spatial interpolation (red) and machine learning methods with PCA-ANN and PCA-Linear for Defect 2 and 3 , respectively.

To deeply investigate the spectral features of replaced radiances for Defect 2-3, Fig. 12 analyzes reflectance spectra by dividing each radiance spectrum in Fig. 11 with the measured solar spectrum of GEMS. In reflectance, measurement noise from calibration processes could be cancelled out by normalizing earth radiance with measured solar irradiance. The Fraunhofer lines originating from the solar spectrum could also be cancelled out, which makes it easy to analyze spectral features of a spectrum without strong absorption lines. In Fig. 12a, spectral discontinuity with spatial interpolation is more obvious at $400 \mathrm{~nm}$ compared to the radiance spectrum in Fig 11a. For Defect 3, Fig. 12b also shows stable features with PCALinear at the defective spectral range around $487 \mathrm{~nm}$. It could also be seen that the measurement noise from the sensor could be successfully incorporated in the replaced spectrum with machine learning methods because the measured reflectance would have noise-like features if the noise is not properly reproduced in the radiance spectrum Consequently, the results indicate that the machine learning methods are good enough to properly reproduce spectrally and spatially continuous features using spectral relations of radiances in the UV/VIS spectrum.

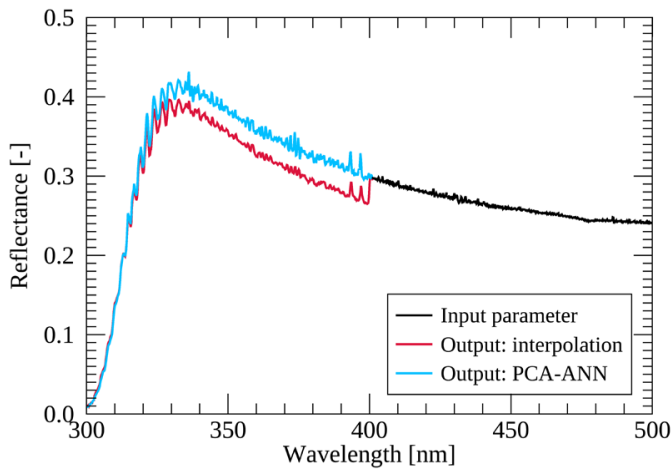

(a)

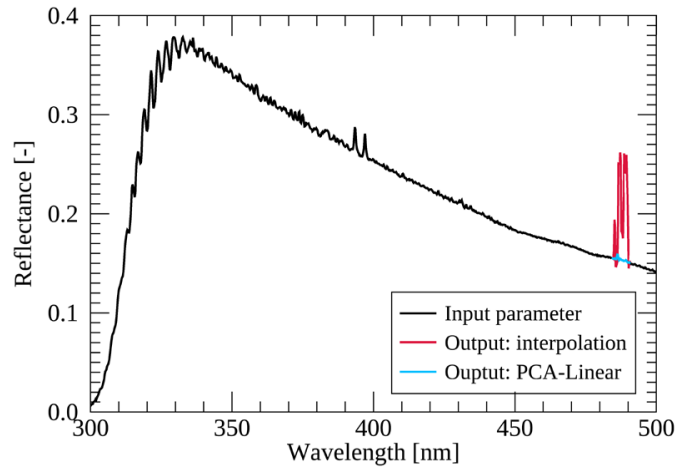

(b)

Figure 12 Same as Fig. 11 for reflectance spectrum affected by bad pixels of (a) Defect 2 and (b) Defect 3. 


\section{Conclusions}

In remote sensing, hyperspectral data in the UV/VIS spectral region are used to retrieve the information on atmospheric gases and aerosol properties. GEMS is an environmental sensor measuring hyperspectral radiances from 300 to $500 \mathrm{~nm}$ in the AsiaPacific region for timely atmospheric monitoring. During the IOT of GEMS, one of calibration issues was found that erroneous values of bad pixels on the detector array are not properly replaced with spatial interpolation, the current operational method of GEMS. It is clear that when the bad pixel area is too large, the spatial interpolation tends to cause high interpolation error especially for a scene having large spatial inhomogeneity (i.e. cloud edges). The high interpolation error of bad pixels could affect to the retrieval process, which causes horizontal discontinuity at a certain latitude for the retrieval of Level 2 products.

To resolve the issue, this study suggests machine learning methods using PCA-ANN and PCA-Linear to fill in the spectral gap caused by bad pixels, denoted as Defect 1-3 in this study. The basic assumption of this approach is that radiances of a spectrum have strong linear and non-linear relations, which could be emulated with the ANN and multivariate linear regression. The spectral range of output radiances corresponds to the wavelengths of bad pixels, while the input radiances correspond to the rest part of a spectrum for Defect 1-3, respectively. Considering that input radiances have strong linear relations, dimensionality reduction with PCA is applied in the pre-processing step to reduce linear relations of input radiances and to increase computational efficiency of training process.

In the results, PCA-Linear model presents smaller prediction error for the defect region having strong linear relations between input and output radiances (Defect 1) or having narrower spectral gap (Defect 3). The PCA-ANN model is better for the output radiances having strong non-linear relations with input radiances (Defect 2). The narrower the spectral range of output radiances is, the smaller the prediction error is because the prediction error of Defect 3 is around $0.5 \%$, while it is around $5 \%$ for Defect 1 and 2. When the trained model is applied to the actual bad pixels, the spectral gap of bad pixels is properly replaced presenting continuous spectral features especially for Defect 2 and 3. The bad pixel replacement with PCA-Linear and spatial interpolation for Defect 1 is almost same considering the narrower spatial gap of Defect 1.

To apply the methods in operation, however, it needs to be updated further to solve the following issues. The machine learning model, especially the PCA-ANN model, becomes highly unstable when measurement characteristics of training and test data significantly change. If measurements have high seasonal dependence, then the time lag between training and test data should be as shorter as possible to guarantee that both data are sampled from an identical population considering the basic premise of ANN. It is empirically found that the time lag between training and test data should not be over two weeks for GEMS which could be technically demanding in operation. Secondly, the radiance at shorter wavelengths (around $325 \mathrm{~nm}$ ) of Defect 2 has high prediction error of over 5\%, which is equivalent to the level of radiometric calibration error of GEMS. To increase prediction accuracy at strong absorption lines, it needs to be updated further.

Considering that the number of bad pixels would increase in operation as did in Ozone Mapping and Profiler Suite (OMPS) (Seftor et al., 2014), an efficient way of replacing bad pixels would be necessary for the long-term operation of GEMS. It is also highly possible that an unexpected issue could occur such as the row-anomaly of Ozone Monitoring Instrument (OMI) 
(Schenkeveld et al., 2017), machine learning methods suggested in this study can be a useful tool for filling in the spectral gap and increasing the number of data reserving measurement characteristics of the sensor.

\section{Author contribution}

M.-H.A. conceptualized and supervised the study; Y.L. conducted the research, performed the experiments and prepared the manuscript; M.K. contributed to the editing of the manuscript and developing methodology. M.E. contributed to the preprocessing of raw data.

\section{Competing interests}

320 The authors declare that they have no conflict of interest.

\section{Acknowledgements}

We would like to thank the Environment Satellite Center (ESC) of National Institute of Environmental Research (NIER) for providing GEMS Level 0 data.

\section{Financial support}

325 This research was supported by Basic Science Research Program through the National Research Foundation of Korea(NRF) funded by the Ministry of Education(2018R1A6A1A08025520).

\section{References}

Bajorski, P.: Statistical inference in PCA for hyperspectral images, IEEE J. Sel. Top. Signal Process., 5(3), 438-445, doi:10.1109/JSTSP.2011.2105244, 2011.

330 Bak, J., Baek, K. H., Kim, J. H., Liu, X., Kim, J. and Chance, K.: Cross-evaluation of GEMS tropospheric ozone retrieval performance using OMI data and the use of an ozonesonde dataset over East Asia for validation, Atmos. Meas. Tech., 12(9), 5201-5215, doi:10.5194/amt-12-5201-2019, 2019.

Boersma, K. F., Eskes, H. J. and Brinksma, E. J.: Error analysis for tropospheric NO2 retrieval from space, J. Geophys. Res. D Atmos., 109(4) [online] Available from: https://agupubs.onlinelibrary.wiley.com/doi/abs/10.1029/2003jd003962 (Accessed

33520 September 2019), 2004.

Boldrini, B., Kessler, W., Rebner, K. and Kessler, R.: Hyperspectral imaging: a review of best practice, performance and pitfalls for inline and online applications, J. Near Infrared Spectrosc., 20(5), 438, doi:10.1255/jnirs.1003, 2012. 
https://doi.org/10.5194/amt-2022-37

Preprint. Discussion started: 17 February 2022

(c) Author(s) 2022. CC BY 4.0 License.
Atmospheric

Measurement

Techniques

Discussions

Burger, J.: Replacement of Hyperspectral Image Bad Pixels, NIR news, 20(7), 19-21, doi:10.1255/nirn.1151, 2009.

Choi, H., Liu, X., Gonzalez Abad, G., Seo, J., Lee, K.-M. and Kim, J.: A Fast Retrieval of Cloud Parameters Using a Triplet

of Wavelengths of Oxygen Dimer Band around 477 nm, Remote Sens., 13(1), 152, doi:10.3390/rs13010152, 2021.

Cybenko, G.: Approximation by superpositions of a sigmoidal function, Math. Control. Signals, Syst., 2(4), 303-314, doi:10.1007/BF02551274, 1989.

Dorvlo, A. S. S., Jervase, J. A. and Al-Lawati, A.: Solar radiation estimation using aritificial neural networks, Appl. Energy, 71(4), 307-319, doi:10.1016/S0306-2619(02)00016-8, 2002.

345 Fischer, A. D., Downes, T. V. and Leathers, R.: Median spectral-spatial bad pixel identification and replacement for hyperspectral SWIR sensors, in Algorithms and Technologies for Multispectral, Hyperspectral, and Ultraspectral Imagery XIII, vol. 6565, edited by S. S. Shen and P. E. Lewis, p. 65651E, SPIE., 2007.

Gewali, U. B., Monteiro, S. T. and Saber, E.: Machine learning based hyperspectral image analysis: A survey, arXiv, 2018.

Goetz, A. F. H., Vane, G., Solomon, J. E. and Rock, B. N.: Imaging spectrometry for earth remote sensing, Science (80-. ).,

228(4704), 1147-1153, doi:10.1126/science.228.4704.1147, 1985.

Hedelt, P., Efremenko, D. S., Loyola, D. G., Spurr, R. and Clarisse, L.: Sulfur dioxide layer height retrieval from Sentinel-5 Precursor/ TROPOMI using FP_ILM, Atmos. Meas. Tech, 12, 5503-5517, doi:10.5194/amt-12-5503-2019, 2019.

Hornik, K., Stinchcombe, M. and White, H.: Multilayer feedforward networks are universal approximators, Neural Networks, 2(5), 359-366, doi:10.1016/0893-6080(89)90020-8, 1989.

Howell, S. B.: CCD imaging, in Handbook of CCD Astronomy, pp. 66-101, Cambridge University Press, Cambridge., 2006.

Kang, M., Ahn, M. H., Liu, X., Jeong, U. and Kim, J.: Spectral calibration algorithm for the geostationary environment monitoring spectrometer (Gems), Remote Sens., 12(17), 1-17, doi:10.3390/rs12172846, 2020.

Kieffer, H. H.: Detection and correction of bad pixels in hyperspectral sensors, in Hyperspectral Remote Sensing and Applications, vol. 2821, edited by S. S. Shen, pp. 93-108, SPIE., 1996.

Kim, G., Choi, Y. S., Park, S. S. and Kim, J.: Effect of solar zenith angle on satellite cloud retrievals based on O2-O2 absorption band, Int. J. Remote Sens., 42(11), 4224-4240, doi:10.1080/01431161.2021.1890267, 2021.

Kim, J., Jeong, U., Ahn, M. H., Kim, J. H., Park, R. J., Lee, H., Song, C. H., Choi, Y. S., Lee, K. H., Yoo, J. M., Jeong, M. J., Park, S. K., Lee, K. M., Song, C. K., Kim, S. W., Kim, Y. J., Kim, S. W., Kim, M., Go, S., Liu, X., Chance, K., Miller, C. C., Al-Saadi, J., Veihelmann, B., Bhartia, P. K., Torres, O., Abad, G. G., Haffner, D. P., Ko, D. H., Lee, S. H., Woo, J. H., Chong, H., Park, S. S., Nicks, D., Choi, W. J., Moon, K. J., Cho, A., Yoon, J., Kim, S. kyun, Hong, H., Lee, K., Lee, H., Lee, S., Choi, M., Veefkind, P., Levelt, P. F., Edwards, D. P., Kang, M., Eo, M., Bak, J., Baek, K., Kwon, H. A., Yang, J., Park, J., Han, K. M., Kim, B. R., Shin, H. W., Choi, H., Lee, E., Chong, J., Cha, Y., Koo, J. H., Irie, H., Hayashida, S., Kasai, Y., Kanaya, Y., Liu, C., Lin, J., Crawford, J. H., Carmichael, G. R., Newchurch, M. J., Lefer, B. L., Herman, J. R., Swap, R. J., Lau, A. K. H., Kurosu, T. P., Jaross, G., Ahlers, B., Dobber, M., McElroy, C. T. and Choi, Y.: New era of air quality monitoring from space: Geostationary environment monitoring spectrometer (GEMS), Bull. Am. Meteorol. Soc., 101(1), E1-E22, doi:10.1175/BAMS-D-18-0013.1, 2020. 
https://doi.org/10.5194/amt-2022-37

Preprint. Discussion started: 17 February 2022

(c) Author(s) 2022. CC BY 4.0 License.
Atmospheric

Measurement

Techniques

Discussions

Kingma, D. P. and Ba, J. L.: Adam: A method for stochastic optimization, in 3rd International Conference on Learning Representations, ICLR 2015 - Conference Track Proceedings, International Conference on Learning Representations, ICLR. [online] Available from: https://arxiv.org/abs/1412.6980v9 (Accessed 7 May 2021), 2015.

375 Le, T., Liu, C., Yao, B., Natraj, V. and Yung, Y. L.: Application of machine learning to hyperspectral radiative transfer simulations, J. Quant. Spectrosc. Radiat. Transf., 246, 106928, doi:10.1016/j.jqsrt.2020.106928, 2020.

Liu, X., Smith, W. L., Zhou, D. K. and Larar, A.: Principal component-based radiative transfer model for hyperspectral sensors: Theoretical concept, Appl. Opt., 45(1), 201-209, doi:10.1364/AO.45.000201, 2006.

Lo'pez-Alonso, J. M. and Alda, J.: Bad pixel identification by means of principal components analysis, Opt. Eng., 41(9), 2152, doi:10.1117/1.1497397, 2002.

Loyola, D. G., Gimeno García, S., Lutz, R., Argyrouli, A., Romahn, F., Spurr, R. J. D., Pedergnana, M., Doicu, A., Molina García, V. and Schüssler, O.: The operational cloud retrieval algorithms from TROPOMI on board Sentinel-5 Precursor, Atmos. Meas. Tech, 11, 409-427, doi:10.5194/amt-11-409-2018, 2018.

Ludewig, A., Kleipool, Q., Bartstra, R., Landzaat, R., Leloux, J., Loots, E., Meijering, P., Van Der Plas, E., Rozemeijer, N., 385 Vonk, F. and Veefkind, P.: In-flight calibration results of the TROPOMI payload on board the Sentinel-5 Precursor satellite, Atmos. Meas. Tech., 13(7), 3561-3580, doi:10.5194/amt-13-3561-2020, 2020.

Manolakis, D., Pieper, M., Truslow, E., Lockwood, R., Weisner, A., Jacobson, J. and Cooley, T.: Longwave infrared hyperspectral imaging: Principles, progress, and challenges, IEEE Geosci. Remote Sens. Mag., 7(2), 72-100, doi:10.1109/MGRS.2018.2889610, 2019.

390 Marchetti, Y., Rosenberg, R. and Crisp, D.: Classification of Anomalous Pixels in the Focal Plane Arrays of Orbiting Carbon Observatory-2 and -3 via Machine Learning, Remote Sens., 11(24), 2901, doi:10.3390/rs11242901, 2019.

Nwankpa, C., Ijomah, W., Gachagan, A. and Marshall, S.: Activation Functions: Comparison of trends in Practice and Research for Deep Learning, arXiv [online] Available from: http://arxiv.org/abs/1811.03378 (Accessed 8 May 2021), 2018.

Pan, C., Weng, F. and Flynn, L.: Spectral Performance and Calibration of the Suomi NPP OMPS Nadir Profiler Sensor, Earth 395 Sp. Sci., 4(12), 737-745, doi:10.1002/2017EA000336, 2017.

Pan, C., Zhou, L., Cao, C., Flynn, L. and Beach, E.: Suomi-NPP OMPS Nadir mapper's operational SDR performance, IEEE Trans. Geosci. Remote Sens., 57(2), 1015-1024, doi:10.1109/TGRS.2018.2864125, 2019.

Pan, C., Yan, B., Cao, C., Flynn, L., Xiong, X., Beach, E. and Zhou, L.: Performance of OMPS Nadir Profilers' Sensor Data Records, IEEE Trans. Geosci. Remote Sens., 1-9, doi:10.1109/tgrs.2020.3026586, 2020.

400 Rankin, B. M., Broadwater, J. B. and Smith, M.: Anomalous pixel replacement and spectral quality algorithm for longwave infrared hyperspectral imagery, Int. Geosci. Remote Sens. Symp., 2018-July, 4316-4319, doi:10.1109/IGARSS.2018.8517461, 2018.

Schenkeveld, V. M. E., Jaross, G., Marchenko, S., Haffner, D., Kleipool, Q. L., Rozemeijer, N. C., Veefkind, J. P. and Levelt, P. F.: In-flight performance of the Ozone Monitoring Instrument, Atmos. Meas. Tech., 10(5), 1957-1986, doi:10.5194/amt- 
Schläpfer, D., Nieke, J. and Itten, K. I.: Spatial PSF nonuniformity effects in airborne pushbroom imaging spectrometry data, IEEE Trans. Geosci. Remote Sens., 45(2), 458-468, doi:10.1109/TGRS.2006.886182, 2007.

Seftor, C. J., Jaross, G., Kowitt, M., Haken, M., Li, J. and Flynn, L. E.: Postlaunch performance of the Suomi National Polarorbiting Partnership Ozone Mapping and Profiler Suite (OMPS) nadir sensors, J. Geophys. Res. Atmos., 119(7), 4413-4428, doi:10.1002/2013JD020472, 2014.

Taylor, M., Kosmopoulos, P. G., Kazadzis, S., Keramitsoglou, I. and Kiranoudis, C. T.: Neural network radiative transfer solvers for the generation of high resolution solar irradiance spectra parameterized by cloud and aerosol parameters, J. Quant. Spectrosc. Radiat. Transf., 168, 176-192, doi:10.1016/j.jqsrt.2015.08.018, 2016.

Wu, W., Liu, X., Xiong, X., Li, Y., Yang, Q., Wu, A., Kizer, S. and Cao, C.: An Accurate Method for Correcting Spectral

415 Convolution Errors in Intercalibration of Broadband and Hyperspectral Sensors, J. Geophys. Res. Atmos., 123(17), 92389255, doi:10.1029/2018JD028585, 2018.

Zarzalejo, L. F., Ramirez, L. and Polo, J.: Artificial intelligence techniques applied to hourly global irradiance estimation from satellite-derived cloud index, in Energy, vol. 30, pp. 1685-1697, Elsevier Ltd., 2005.

Zhen, Y. and Li, C.: Cross-Domain Knowledge Transfer Using Semi-supervised Classification, Lect. Notes Comput. Sci. 420 (including Subser. Lect. Notes Artif. Intell. Lect. Notes Bioinformatics), 5360 LNAI, 362-371, doi:10.1007/978-3-540-893783_36, 2008.

Zhu, S., Lei, B. and Wu, Y.: Retrieval of hyperspectral surface reflectance based on machine learning, Remote Sens., 10(2), 1-15, doi:10.3390/rs10020323, 2018. 